\title{
COMPETENCIAS CIUDADANAS: EJES PRESENTES EN EL SECTOR ESTUDIANTIL COLOMBIANO
}

\author{
Nely Monterrosa* \\ https://orcid.org/0000-0002-6447-0197
}

RECIBIDO: Agosto 2019 / ACEPTADO: Marzo 2020 / PUBLICADO: Mayo 2020

Como citar: Monterrosa, Nelly (2020). Competencias ciudadanas: ejes presentes en el sector estudiantil colombiano. Telos: revista de Estudios Interdisciplinarios en Ciencias Sociales, 22 (2), Venezuela. (Pp.376-386).

DOI: www.doi.org/10.36390/telos222.09

\section{RESUMEN}

Esta investigación tuvo como objetivo analizar los ejes de las competencias ciudadanas en un sector estudiantil colombiano seleccionado por las características conductuales manifestadas por ellos dentro de la institución donde cursan estudios. Teóricamente se sustentó en planteamientos sobre competencias ciudadanas de Chaux, Lleras y Velázquez (2012), Mockus (2012), Tedesco, Opertti y Amadio (2013), así como con las políticas establecidas por el Ministerio de Educación Nacional (2005), aun en vigencia para efectos de este trabajo. El contexto paradigmático fue positivista cuantitativo, la investigación descriptiva, con diseño no experimental, de campo. La muestra poblacional fue de veintiún (21) estudiantes de básica secundaria de la Institución Educativa Distrital Beatriz Gutiérrez de Vives. Se aplicó un censo y una técnica de observación por encuesta mediante instrumento escalar con alternativas de respuesta Siempre, Casi Siempre, Casi Nunca y Nunca, validado por cinco expertos y con un coeficiente Alfa de Cronbach de 0.86 . Se evidencia que los ejes de las competencias ciudadanas tienen una alta presencia en el sector estudiantil colombiano seleccionado, sin embargo, se proponen algunas acciones para mejorar algunos detalles observados durante el estudio, con las cuales sea posible un proceso de implementación de la convivencia ciudadana para optimizar las relaciones interpersonales entre los estudiantes en la institución educativa, dado que allí transcurre de forma compartida gran parte de su tiempo diariamente.

Palabras clave: Ejes de la Competencia Ciudadana, Convivencia, Participación, Pluralismo.

\section{Citizen competences: axes present in the colombian student sector}

\begin{abstract}
This research aimed to analyze the axes of citizen competencies, in a selected Colombian student sector by the behavioral characteristics expressed by them in the institution where they study. Theoretically, it was based on approaches on citizen competences of Chaux, Lleras and
\end{abstract}

\footnotetext{
* Magister en Informática Educativa. Correo electrónico: nelymonterrosa@hotmail.com
} 
Velázquez (2012), Mockus (2012), Tedesco, Opertti and Amadio (2013), as well as the policies established by the Ministry of National Education (2005), still valid for the purposes of this work. The paradigmatic context was quantitative positivist, the research was descriptive, with nonexperimental, field design. The population sample was twenty-one (21) high school students from the Beatriz Gutiérrez de Vives District Educational Institution. A census and a survey observation technique was applied using a scalar instrument with response alternatives: Always, Almost Always, Almost Never and Never, validated by five experts and with a Cronbach's alpha coefficient of 0.86 . It is evident that the axes of citizen competencies have a high presence in the selected Colombian student sector, however, some actions are proposed to improve some details observed during the study, with which it is possible to implement a process of citizen coexistence to optimize interpersonal relationships between students in the educational institution, since there they spend a part of their daily time together.

Keywords: Axis of Citizen Competition, Coexistence, Participation, Pluralism.

\section{Introducción}

La ciudadanía es un término imbuido en un contexto caracterizado por la controversia, básicamente por causa de la variedad de escenarios con los cuales se involucra, como lo son los aspectos políticos, sociales, educativos, económicos y culturales de la sociedad, así como lo es la subjetividad personal de quienes conviven en ella y sus formas de interpretarla (Pachajoa Londoño, 2015). Al estar relacionado con distintos ámbitos, ha sufrido varias modificaciones con el paso del tiempo, como se plantea a continuación.

Durante los primeros años del siglo XIX la sociedad liberal introdujo el término de educación cívica como basamento del desarrollo democrático de las naciones (Gros y Contreras, 2006), siendo definido por Musalem (2016) como un procedimiento de promoción del conocimiento y la comprensión de la normativa bajo la cual se regula "...la vida social y la formación de valores y actitudes que permiten al individuo integrarse a la sociedad y participar en su mejoramiento" (p. 6). Su evolución conllevó a replantear la concepción y simbología de la ciudadanía, trayendo como consecuencia el ajuste de la expresión "educación cívica" hacia la actual locución de "competencias ciudadanas".

Se concibe de esta manera la formación ciudadana como una educación cívica relacionada con un ejercicio participativo a través de cual se puede reflexionar y argumentar respecto a acciones políticas conducentes a mejorías en el destino de la población (García Guitián, 2006). En general, se parte del principio básico relacionado con el derecho a la información del ciudadano. De conformidad con lo expresado por Gumucio Dragon (2006), el problema a enfrentar puede resolverse a través de la comunicación. A su parecer, la competencia ciudadana, "...en la medida en que potencia las voces de los sujetos del desarrollo, fortalece sus organizaciones sociales, y facilita procesos de participación social a través del diálogo y del debate" (p.10)

Como resultado, las competencias ciudadanas superan sus limitantes teóricas, consistiendo para el estudiante en un "saber hacer" con ellas, es decir, comportándose como personas informadas y participativas (López, 2008), prevaleciendo de este modo la formación académica, al abarcar habilidades y actitudes con las cuales podrán actuar, participar, asociarse, 
organizarse, intercambiar opiniones, emprender acciones colectivas y expresar sus opiniones, entre otras acciones relacionadas con conductas socialmente aceptadas.

\section{Ejes de las Competencias Ciudadanas}

Cuando se habla de competencias ciudadanas se hace alusión a todos los procesos necesarios para lograr la convivencia pacífica de la sociedad, lo cual involucra de manera especial los aspectos educativos. En este sentido, uno de los grandes retos para la educación en la actualidad es lograr que la comunidad vinculada con esta actividad discuta de forma democrática y abiertamente la realidad que se esté evidenciando en la vida escolar y familiar, a fin de estar en capacidad de alcanzar acuerdos respecto a la formación moral de los estudiantes.

Esta situación constituye un proceso, como se mencionó con anterioridad, el cual es denominado por el psicólogo estadounidense Lawrence Kohlberg (2005, citado por Caro Samada, Ahedo Ruiz y Esteban Bara, 2018), como la "Escuela Justa", espacio donde adquiere importancia la temática referida a los ejes de las competencias ciudadanas y su presencia en los documentos curriculares.

Según Tedesco, Opperti y Amadio (2013), estos temas generalmente se piensan como (a) una respuesta pedagógica para relacionar contenidos a través de diferentes ámbitos disciplinares, (b) promover un enfoque multidisciplinario o interdisciplinario, (c) ampliar y enriquecer la oferta curricular sin sobrecargar el currículo, (d) facilitar el aprendizaje cooperativo, (e) tratar cuestiones de relevancia para la vida de los estudiantes y en varios casos,(f) para contribuir al desarrollo de las competencias clave.

De manera complementaria, debe reconocerse el establecimiento de la política transversal del Ministerio de Educación Nacional (2005), para el currículo educativo con miras al desarrollo de las competencias ciudadanas, tanto en la escuela como en el hogar. Una política caracterizada por haber sido concertada entre docentes, padres de familia, instituciones de educación y profesionales de diversas disciplinas, resultando de ello un trabajo integral y beneficioso para las partes involucradas.

En ese sentido, cabe recordar que las competencias ciudadanas comprenden tres ejes temáticos: (a) la construcción de la convivencia y la paz para un mejor vivir, (b) la participación y responsabilidad democrática, (c) la pluralidad, identidad y valoración de las diferencias, conocidos también como estándares básicos, de conformidad con los planteamientos de Fuentes y Pérez (2019) En el primero se incluyen las habilidades de los colombianos para mediar y llegar a acuerdos y pactos de convivencia, con el objetivo de resolver los conflictos de forma pacífica.

El segundo eje tiende hacia la configuración de una sociedad comprometida, defensora del bien común y respetuosa de las diferencias; mientras el tercero, abarca la formación de unos ciudadanos seguros de sí mismos, capaces de analizar y aportar en procesos colectivos de participación, responsabilidad democrática y pluralidad. Con ello se aspira a que los estudiantes de los planteles educativos aprendan, entre otros aspectos, a crear campañas de conservación de los recursos naturales así como a organizar proyectos colectivos y solidarios, potenciando las habilidades comunicativas como vehículo de construcción de identidad y resolución de conflictos.

De acuerdo con lo anteriormente expuesto, han surgido sugerencias referidas a la promoción del trabajo colaborativo de docentes pertenecientes a diferentes disciplinas en la 
planificación de las actividades pedagógicas con la cuales se posibilite reforzar la responsabilidad colectiva para el aprendizaje de los estudiantes en la educación secundaria, aprovechando la transversalidad de esos temas, a sabiendas que estos están relacionados con los ejes de las competencias ciudadanas, abarcando una gran variedad de tópicos y formaciones, como lo son, por ejemplo la educación en valores, la educación cívica y ciudadana, la educación para la salud, la educación para los derechos humanos, las TIC o la igualdad de género.

Para efectos de este trabajo, se fija posición con las políticas establecidas por el Ministerio de Educación Nacional (2005), las cuales están orientadas al desarrollo de las competencias ciudadanas tanto en la escuela como en el hogar, teniendo como objetivo la creación de una identidad y la valoración de las diferencias, con lo cual puede concretarse, a su vez, la formación de ciudadanos seguros de sí mismos, capaces de analizar y aportar en procesos colectivos de participación, responsabilidad democrática y pluralidad.

Los ejes de las competencias ciudadanas generalmente se conciben como una respuesta pedagógica para relacionar contenidos a través diferentes ámbitos disciplinares, promover un enfoque multidisciplinario 0 interdisciplinario, ampliar y enriquecer la oferta curricular, facilitar el aprendizaje cooperativo, tratar cuestiones de relevancia para la vida de los estudiantes y en ocasiones para contribuir al desarrollo de las propias competencias ciudadanas. De ahí la importancia de lograr que en el ámbito educativo se promueva el trabajo colaborativo entre docentes de diferentes disciplinas con el fin de planificar las actividades pedagógicas y reforzar la responsabilidad colectiva entre los estudiantes.

\section{Convivencia}

La convivencia es definida por Chaux, Lleras y Velázquez (2012), como la habilidad que poseen los ciudadanos para vivir en paz y de forma constructiva, sin agresiones y favoreciendo a los entes involucrados, utilizando mecanismos pacíficos para hacer valer sus derechos y los derechos de terceros, procurando acuerdos apoyados en las normas vigentes, acudiendo a instancias de regulación y mediación institucional cuando sea necesario. Una convivencia pacífica que incluye también las relaciones con el medio ambiente, con el propósito de garantizar recursos naturales a las nuevas generaciones.

Casi diez años antes, ya López (2008), definía la convivencia como una dimensión fundamental de la ciudadanía, lo cual permite percibir su relación con el marco del respeto, la promoción, la defensa de los derechos humanos y la valoración de las diferencias tanto locales como globales. Por su parte, unos años antes, Mockus y Corso (2003), expresaban que la convivencia permite alcanzar una relación entre factores diversos, como lo son: el acatamiento voluntario de la ley, el cumplimiento de los acuerdos, el conocimiento y la aplicación de los mecanismos democráticos para lograr vivir en armonía.

Todo ser humano, en general, vive en sociedad, por tanto, se relaciona con otros. Una realidad factible de ser cumplida de una forma u otra, a través de la cual debe compartir con el resto de las personas que habitan en el mismo ámbito, siendo importante la actitud de cada uno de respetar al otro, incluso en su requerimiento de contar esporádicamente con espacios de intimidad, para lograr una convivencia agradable.

Una convivencia demostrativa de la inteligencia emocional del individuo, basada en ese respeto mutuo. Su relevancia se centra en acciones que permitan lograr un ambiente ameno a 
los demás, sin perjudicar a nadie, independientemente del espacio donde se encuentren, sea el colegio, la universidad, el hogar, el trabajo, es decir, donde quiera que pueda desempeñarse el individuo en sociedad.

\section{Participación}

De acuerdo con Chaux, Lleras y Velázquez (2012), la participación se define, en general, como el deber que tiene el ciudadano de asistir a los comicios electorales, los cuales deben tener participación activa y crítica de todos, para que la construcción de acuerdos o tomas de decisiones, tanto macros como micros, estén reguladas por todos los entes de la democracia y sea garantizada la transparencia de sus elecciones. En ese sentido, se trata de un ejercicio donde se invita a la participación de todos, debido a que es una práctica viable en todos los ámbitos del ciudadano, como los son, por ejemplo, una organización, una institución educativa 0 una familia.

La idea es conseguir acuerdos, decisiones y acciones por voto directo de los involucrados, utilizando para ello el diálogo y el consenso. Partiendo de las políticas de competencias ciudadanas, se trata de instaurar procesos democráticos tanto en la escuela como en la sociedad. A nivel educativo, una práctica común es la selección de consejeros estudiantiles, para lo cual deben comprender que sus acciones deben estar regidas por el respeto de los derechos humanos básicos, mientras se forman ciudadanos capaces de defender sus ideas con inteligencia, de modo argumentativo, evitando la manipulación o la coerción.

Como parte de las políticas implementadas por el Estado neogranadino a mediados de la década del 2000 , se reconoce como parámetro oficial que la participación esté orientada hacia "la toma de decisiones en diversos contextos, teniendo en cuenta que dichas decisiones deben respetar, tanto los derechos fundamentales de los individuos, como los acuerdos, las normas, las leyes y la Constitución que rigen la vida en comunidad" (Ministerio de Educación Nacional, 2004, p. 12).

Por su parte, Dalla Corte (2011), alude una visión normativa, desde la cual el término de participación se puede restringir a los casos representativos de alguna respuesta, individual 0 colectiva, de la sociedad ante una convocatoria realizada por parte del gobierno en aquellos espacios institucionales designados por ellos. Al citar a Balbis (Dalla Corte, 2011), retoma de él su concepción para complementar la suya, quien la define "como toda forma de acción colectiva que tiene por interlocutor a los Estados y que intenta -con éxito o no- influir sobre las decisiones de la agenda pública" (p.115)

Los autores concuerdan respecto a la necesidad de asistir a todos los eventos en los cuales el ciudadano tenga el deber de realizar una selección de tipo político, para que así se pueda garantizar la normal transición de la democracia en todos los sectores del poder público y también se pueda influir en las decisiones que de una u otra forma afectan al gobierno del país en donde se encuentre localizado el individuo. Se entiende de este modo la participación como el proceso donde las personas aportan sus ideas, las cuales pueden o no ser compartidas por los demás.

Sin embargo, se hace necesario que en la sociedad todas las personas puedan ser partícipes de las acciones lideradas en su contexto. Ser miembro activo de un grupo genera espacios de interacción donde se comparten ideas que contribuyen significativamente al enriquecimiento de los conocimientos para reformularlos, afianzarlos 0 modificarlos, al 
comprender un concepto que no se tenía claro. De conformidad con estos planteamientos, la democracia exige personas participativas, para estar en capacidad de coadyuvar en la construcción de las políticas de su estado o del contexto en el que esté inmerso.

\section{Pluralidad}

Con respecto a este término, Chaux, Lleras y Velázquez (2012), manifiestan que es la capacidad de identificar y respetar las diferencias y semejanzas entre los demás y el individuo. También comprende la capacidad de rechazar situaciones de exclusión o discriminación en la sociedad. El acatamiento de la ciudadanía necesariamente implica una comprensión de las costumbres, valores, tradiciones, formas de comunicación del lugar donde se convive. Todas estas características constituyen el fundamento de una sociedad pluralista.

Sin embargo, cada uno de los ejes de las competencias ciudadanas tienen un límite, es decir, la pluralidad y la valoración de diferentes consensos personales no pueden conllevar a que se tolere a quienes vulneran algún tipo de derechos; por ende, en la pluralidad hay barreras regidas por leyes en las cuales se especifican los derechos de todos los ciudadanos que conforman la sociedad en estudio.

Este planteamiento posibilita caracterizar la pluralidad como el ejercicio de la ciudadanía para reconocer a otros ciudadanos, lo cual significa ser tolerante con respecto a los pensamientos de terceros, sus creencias, personalidades, culturas, razas, así como reconocer cada diferencia personal que se presenten en el ámbito donde haya una convivencia, entendiendo por convivencia la capacidad de los ciudadanos de vivir en paz y de forma constructiva, como lo describen Chaux, Lleras y Velázquez (2012).

Desde la perspectiva de Cabrero (2008, p.13), al hablar de pluralidad se expresa la necesidad de pensar en dos ejes fundamentales: lucha política y lucha desde la epistemología, manifestando que "Para poder alcanzar una "ética intercultural" se precisa, sobre todo, reconocer al "Otro" y valorar el diálogo", agregando, al citar a Macas (Cabrero, 2008, p.10), "Desde otros aportes, desde otras existencias, desde otros pueblos". Se comprende así que la pluralidad le permite al individuo adoptar costumbres y tradiciones de otros pueblos, para hacerlos suyos y considerarlos como propios, pero cuando entra en el terreno de las convicciones políticas, sociales y religiosas, es posible observar la diversidad de pensamientos existentes, los cuales de una u otra forma deben ser respetados.

\section{Aspectos Metodológicos}

El estudio es descriptivo, no experimental, de campo. De acuerdo con Arias (2012), Tamayo y Tamayo (2014), Hernández Sampieri, Fernández Collado y Baptista Lucio (2015),aspira a puntualizar las cualidades, características significativas de personas, grupos e incluso de cualquier otro fenómeno o evento expuesto a un análisis, es decir, que este tipo de investigación intenta tanto medir como agrupar información de forma independiente o conjunta sobre las nociones o variables observadas, sin indicar cómo se relacionan; siendo no experimental porque sólo se observa el fenómeno objeto de estudio en su contexto real para describirlo, sin ninguna manipulación por parte de quien desarrolla la indagación.

Respecto a la población, se ubicó un grupo escolar donde fuese posible contar con una permisología previa para consultarlos. De este modo quedó conformada por veintiún (21) estudiantes de ambos sexos, pertenecientes al grado noveno $9^{\circ}$ de básica secundaria de la 
Institución Educativa Distrital Beatriz Gutiérrez de Vives de la Ciudad de Santa Marta, caracterizándose como finita, conformando por su disposición y accesibilidad un censo poblacional (Hernández et al, 2015).

En relación con la selección de esta institución en particular, se justifica por la existencia de algunas características particulares, como su ubicación y reportes de conducta violenta manifestados por los jóvenes durante su convivencia en horas de clase. Es un colegio que atiende a un sector poblacional calificado como estrato uno, donde habitan familias con condiciones económicas limitadas, con vivencias de robo, consumo de drogas y conductas agresivas, reflejándose esta agresividad entre la comunidad escolar. Por ello, se decidió analizar en ellos las competencias ciudadanas como mecanismo que posibilite implementar un proceso para mejorar su comportamiento mediante el rescate de valores como el respeto.

Para desarrollar el estudio, fue seleccionada como técnica la observación mediante encuesta, definida por Arias (2012), como aquella que pretende obtener información suministrada por un grupo o muestra de sujetos acerca de un tema particular. Según Tamayo y Tamayo (2014), es una forma concreta de la técnica de la observación, constituida por preguntas, formuladas por escrito, que generalmente no requieren la presencia del investigador para su aplicación. Por ello se diseñó un instrumento escalar de 15 ítems, repartidos de modo equitativo entre cada tipo de competencia considerada, con alternativas de respuesta Siempre, Casi Siempre, Casi Nunca y Nunca, validado por cinco expertos y una confiabilidad basada en la estimación del coeficiente Alfa de Cronbach, cuyo resultado fue de 0,86 , utilizándose para el análisis de los resultados el siguiente baremo.

Tabla 1.

Baremo de Categorización de las Repuestas

\begin{tabular}{cc}
\hline Nivel & Rango \\
\hline Alta presencia de la competencia & $4.00-5.00$ \\
Presencia media de la competencia & $3.00-3.99$ \\
Baja presencia de la competencia & $1.00-2.99$ \\
\hline
\end{tabular}

Fuente: Elaboración propia.

\section{Resultados}

Los resultados de la aplicación del instrumento a la población objeto de estudio fueron tabulados para ser analizados, empleando la distribución de frecuencias para las alternativas de respuesta seleccionadas por cada sujeto, así como la estimación de la media aritmética, que permite establecer la tendencia obtenida para compararla con el baremo diseñado para este fin a partir del modelo propuesto por Hernández et al., (2015). 
Tabla 2.

Ejes de Competencias Ciudadanas

\begin{tabular}{lccccccccc}
\hline $\begin{array}{l}\text { Opciones de } \\
\text { Respuesta }\end{array}$ & Siempre & $\begin{array}{c}\text { Casi } \\
\text { Siempre }\end{array}$ & $\begin{array}{c}\text { Casi } \\
\text { Nunca }\end{array}$ & Nunca & Promedios \\
\hline Ejes de Competencia & F & $\%$ & F & $\%$ & F & $\%$ & F & $\%$ & Ítems \\
\hline \multirow{2}{*}{ Convivencia } & 7 & 33 & 10 & 48 & 3 & 14 & 1 & 5 & \\
& 10 & 48 & 11 & 52 & 0 & 0 & 0 & 0 & 4.7 \\
& 11 & 52 & 4 & 19 & 5 & 24 & 1 & 5 & \\
& 6 & 28.5 & 6 & 28.5 & 5 & 24 & 4 & 19 & \\
Participación & 8 & 38 & 5 & 24 & 8 & 38 & 0 & 0 & 4.4 \\
& 3 & 14 & 11 & 52 & 5 & 24 & 2 & 10 & \\
& 7 & 33 & 6 & 29 & 1 & 5 & 7 & 33 & \\
Pluralidad & 4 & 19 & 9 & 43 & 5 & 24 & 3 & 14 & 4.4 \\
& 10 & 48 & 3 & 14 & 1 & 5 & 7 & 33 & \\
\hline
\end{tabular}

\section{Promedio General}

Fuente: Elaboración propia.

Para cada uno de los ejes se realizaron tres interrogantes básicas, como se muestra en la Tabla2. Los resultados evidencian que respecto a la convivencia, la mayoría de los encuestados construye relaciones pacíficas en su escuela para mejorar la convivencia entre compañeros, representados por $33 \%$ de respuestas siempre y $48 \%$ por casi siempre; $19 \%$ manifestó no realizar ese tipo de actividad casi nunca (14\%) y nunca $(5 \%)$. Por otro lado, $48 \%$ expresó evitar siempre acciones que causen sufrimiento a otras personas, en tanto $52 \%$ lo hace casi siempre, permitiendo percibir que todos procuran, de una u otra forma, prevenir el perjuicio hacia terceros.

Respecto a la construcción de acuerdos entre compañeros para lograr la convivencia, $52 \%$ seleccionó la opción siempre y $19 \%$ casi siempre, llamando la atención que casi la cuarta parte de ellos (24\%) casi nunca lo hace, mientras $5 \%$ nunca realiza este tipo de acción. A nivel general, el promedio de las respuestas obtenidas refleja un valor de 4.7, demostrando una alta presencia de la competencia en los sujetos contactados de conformidad con el baremo de categorización de respuestas preparado para efectos de este análisis.

De este modo se observa que, a pesar de las características que conllevaron a la selección de esta institución escolar, los resultados muestran similitud con la teorización de Chaux, Lleras y Velázquez (2012), referida a la habilidad de los ciudadanos para vivir en paz y 
de forma constructiva, sin agresiones y favoreciendo a los entes involucrados, utilizando mecanismos pacíicos para los derechos de cada uno, como los acuerdos, procurando establecer relaciones respetosas (López, 2008), enmarcadas en los aspectos legales (Mockus y Corso, 2003), para lograr vivir en armonía.

En referencia a la participación, hubo un reparto equitativo entre quienes manifestaron liderar siempre $(28.5 \%)$ y casi siempre $(28.5 \%)$ iniciativas democráticas en su medio escolar, con criterios de justicia, solidaridad y equidad; aunque $24 \%$ (casi la cuarta parte de los encuestados) escogió la opción casi nunca y $19 \%$ nunca. Al preguntar si participan en iniciativas democráticas en su medio escolar como forma de promover la defensa de los derechos civiles, las respuestas fueron siempre y casi siempre para $38 \%$ y $24 \%$, respectivamente, pero otro $38 \%$ declaró casi nunca tomar parte de esas actividades.

Como complemento al análisis de este eje, se indagó sobre la participación en la planeación de acciones mediante las cuales se posibilita aliviar la situación de personas que se encuentran en desventaja, obteniéndose como respuestas $14 \%$ siempre, $52 \%$ casi siempre, $24 \%$ casi nunca y $10 \%$ nunca. El promedio de respuestas de este eje es de 4.4 , interpretable como alta presencia de esta competencia en el grupo de sujetos de investigación contactados.

Los datos permiten percibir el cumplimiento de la teoría de Chaux, Lleras y Velázquez (2012), respecto a la necesidad de participar activamente en la consecución de acuerdos mediante el diálogo y el consenso, regidos por el respeto de los derechos humanos básicos y el aporte de ideas como ejercicio democrático.

Finalmente, en relación con la pluralidad, se preguntó sobre el rechazo hacia situaciones de discriminación presentadas en la escuela, ante lo cual $33 \%$ respondió siempre y $29 \%$ casi siempre, en tanto $5 \%$ optó por la expresión casi nunca y 33\% nunca, llamando la atención que los extremos de las respuestas presentadas obtuvieron igual valoración. Sin embargo, al sumar los datos positivos, conforman una mayoría en la tendencia de opciones apartadas. Seguidamente, se indagó si intervienen como mediadores para evitar las acciones de exclusión social entre compañeros, observándose que $19 \%$ y $43 \%$ lo hacen siempre o casi siempre, respectivamente, ante $24 \%$ (casi nunca) y $14 \%$ (nunca) de opciones inclinadas a una menor frecuencia.

Referente a si rechazan cualquier discriminación a compañeros que tengan otra orientación sexual, $48 \%$ expresó siempre, $14 \%$ casi siempre, $5 \%$ casi nunca y $33 \%$ nunca, permitiendo percibir aspectos relacionados con su nivel de tolerancia. Conforme los datos expresados en la tabla contentiva de los resultados, este eje obtuvo un promedio de 4.4, reflejando, al igual que los dos anteriores, una alta presencia de la competencia, coincidiendo con Chaux, Lleras y Velázquez (2012), quienes definen la pluralidad como la capacidad de identificar y respetar las diferencias y semejanzas entre los demás y el individuo, incluyendo de igual modo la capacidad de rechazar situaciones de exclusión o discriminación, comprendiendo los valores, tradiciones y formas de comunicación dentro del espacio de convivencia al reconocer al otro (Cabrero, 2008), con su diversidad de pensamientos.

Al estimar el valor general de los tres tipos de ejes analizados resulta de 4.5 , ratificando su alta presencia en los estudiantes encuestados. Su contraste con los planteamientos teóricos que sustentan la variable competencias ciudadanas, manifiesta coincidencia con la conceptualización de Mockus (2012), Chaux, Lleras y Velázquez (2012), quienes la definen como la habilidad de los ciudadanos para manifestar su capacidad de vivir constructivamente y 
en paz, evitando las agresiones al utilizar mecanismos pacíficos para hacer valer los derechos de cada quien, llegando a acuerdos apoyados en las normas vigentes 0 a través de mediación institucional cuando sea necesario.

\section{Conclusiones}

La información recopilada para dar alcance al objetivo analizar los ejes de las competencias ciudadanas en el sector estudiantil colombiano, permite concluir que hay percepción adecuada sobre los mismos, constituyendo una fortaleza tanto para la institución educativa como para los núcleos familiares involucrados con los participantes del estudio. Sin embargo, no pueden pasarse por alto los aspectos donde las respuestas de menor frecuencia ponen de manifiesto la necesidad de atender tendencias que pueden convertirse en elementos negativos si no ponen en práctica algunas acciones correctivas de inmediato.

Como recomendación, puede implementarse a nivel local con un ciclo de charlas o de talleres con los cuales sea factible adquirir los conocimientos necesarios para fortalecer la acción pedagógica-comunitaria mientras se internalizan los ejes de las competencias ciudadanas descritos y lograr la transformación requerida en el ámbito educativo. Este accionar se propone como lineamiento de implementación de la convivencia ciudadana para optimizar las relaciones interpersonales entre los estudiantes en la institución educativa, dado que allí transcurre de forma compartida gran parte de su tiempo diariamente, pero de igual manera, los resultados se consideran de utilidad como insumos para generar políticas públicas vinculantes.

Esto requiere, a su vez, de varios aspectos simultáneos. En principio, debido a la necesidad de incorporar esas acciones a la planificación académica, exige un compromiso real por parte de directiva, docentes y alumnado, pudiendo proponer jornadas de integración entre docente y estudiante en días especiales, capacitar a la comunidad sobre los valores, con énfasis en el respeto, la comunicación y la tolerancia; así como el desarrollo de campañas preventivas sobre el bullying, considerando que éste puede manifestarse a nivel personal, pero también suele hacerse presente a través de las redes sociales, para disminuir su uso mediante campañas de sensibilización sobre la sana convivencia.

\section{Referencias Bibliográficas}

Arias, Fidias (2012). El Proyecto de Investigación (sexta edición). Editorial Episteme. Venezuela.

Cabrero, Ferran (2008). Diversidad, Pluralismo e Interculturalidad. Extraído dehttp://auditoriaalademocracia.org/web/wp-content/uploads/2013/10/049.cabrero-2008.-Diversidad-pluralismo-e-interculturalidad.pdfconsulta: 17/07/19.

Caro Samada, Carmen., Ahedo Ruiz, Josu y Esteban Bara, Francisco (2018). La Propuesta de Educación Moral de Kohlberg y su Legado en la Universidad: Actualidad y Prospectiva. Revista Española de Pedagogía. Año LXXVI, №269. España (Pp.85-100).

Chaux, Enrique., Lleras, Juanita y Velázquez, Ana María (2012). Competencias ciudadanas: de los estándares al aula. Una propuesta de integración de las áreas académicas. Universidad de los Andes.Colombia.

Dalla Corte, Gabriella (2011). Historias, Indígenas, Nación y Estado en el Bicentenario de la Independencia de la República del Paraguay (1811-2011). Edicions Universitat Barcelona. España. 
Fuentes, Luz y Perez, Libia (2019). Convivencia Escolar: una Mirada desde las Familias. Revista Telos. Volumen 21, No1. Venezuela (Pp.61-85). DOI: https://doi.org/10.36390/telos211.05

García Guitián, Elena (2006). La Educación Cívica como Educación Política. Fundación Manuel Giménez Abad de estudios Parlamentarios y del estado Autonómico. España.

Gros, Begoña y Contreras, David (2006). La alfabetización digital y el desarrollo de competencias ciudadanas. Revista Iberoamericana de Educación. N42. España (Pp. 113-116).

Gumucio Dagron, Alfonso (2006). Tiempo de Milagros: tres retos de la comunicación para el cambio social.

Extraído

dehttp://www.c3fes.net/\%28d\%29comciudyder\%28f\%29.htm consulta: 14/01/19.

Hernández Sampieri, Roberto., Fernández Collado, Carlos y Baptista Lucio, Pilar (2015). Metodología de Investigación. Editorial McGraw-Hill. México.

López, Leopoldo (2008). Convivencia y Seguridad Ciudadana: Hacia estrategias integrales de prevención y control de la violencia y la delincuencia para las ciudades venezolanas. Editorial Santillana. Venezuela.

Ministerio de Educación Nacional (2004). Formar para la Ciudadanía... jsí es posible! Lo que necesitamos saber y saber hacer. Cartilla Guía 6. MEN -Bogotá. Colombia.

Ministerio de Educación Nacional (2005). Foro Educativo Nacional de Competencias Ciudadanas. MEN-Bogotá. Colombia.

Mockus, Antanas (2012). Una lección de cultura ciudadana con Antanas Mockus. Extraído dehttps://periodismopublico.com/Una-leccion-de-Cultura-Ciudadanaconsulta: $18 / 08 / 2019$.

Mockus, Antanas y Corso, Jimmy (2003). Cumplir para Convivir. Factores de Convivencia y su Relación con Normas y Acuerdos. Universidad Nacional de Colombia. Instituto de Estudios Políticos y Relaciones Internacionales. Colombia.

Musalem, Nelly (2016). Manual de Educación Cívica. UQBAR Editores. Chile.

Pachajoa Londoño, Alejandro (2015). Prólogo. En: González Hernández, Dory Luz., Rojas Claros, Armando., García Rodríguez, Gustavo Octavio., González Hernández, Chris Aleydi., Chaustre Avendaño, Álvaro., Vásquez, Jaime Enrique., Rodríguez, Meybel Dayana y Álvarez Castro, Diego Fernando (2015). La Ciudadanía en Controversia: Análisis y Conceptualización de las Competencias Ciudadanas. Fundación Universitaria Los Libertadores. Colombia.

Tamayo y Tamayo, Mario (2014). El Proceso de la Investigación Científica. Limusa. México.

Tedesco, Juan Carlos., Opertti, Renato y Amadio, Massimo (2013). Porqué Importa Hoy el Debate Curricular. IBE Working Papers on Curriculum Issues $N^{\circ} 10$.UNESCO Oficina Internacional de Educación. Suiza (Pp. 1-23). 